\title{
Correlation functions in the Schwarzian theory
}

\author{
Vladimir V. Belokurov ${ }^{a, b}$ and Evgeniy T. Shavgulidze ${ }^{a}$ \\ ${ }^{a}$ Lomonosov Moscow State University, \\ Leninskie gory 1, Moscow, 119991, Russia \\ ${ }^{b}$ Institute for Nuclear Research of the Russian Academy of Sciences, \\ 60th October Anniversary Prospect 7a, Moscow, 117312, Russia \\ E-mail: vvbelokurov@yandex.ru, shavgulidze@bk.ru
}

ABSTRACT: A regular approach to evaluate the functional integrals over the quasi-invariant measure on the group of diffeomorphisms is presented. As an important example of the application of this technique, we explicitly evaluate the correlation functions in the Schwarzian theory.

KEYwords: Field Theories in Lower Dimensions, AdS-CFT Correspondence, Integrable Field Theories

ARXIV EPRINT: 1804.00424 


\section{Contents}

1 Introduction 1

2 Preliminaries 3

3 Correlation functions $\quad 7$

3.1 Mean value of $\varphi^{\prime} \quad 7$

3.2 Two-point correlation function 9

$\begin{array}{lll}3.3 & \text { N-point correlation functions } & 14\end{array}$

4 Concluding remarks $\quad 15$

$\begin{array}{ll}\text { A A quasi-invariant measure on the group of diffeomorphisms } & 15\end{array}$

$\begin{array}{ll}\text { B Proof of the basic formula } & 18\end{array}$

$\begin{array}{ll}\text { C Properties of the function } \mathcal{E}_{\sigma}(u, v) & 19\end{array}$

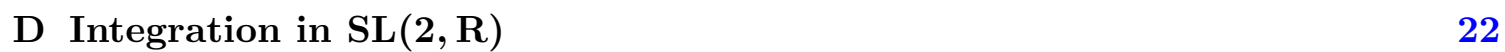

\section{Introduction}

The Schwarzian theory is the basic element of various physical models including the SYK model and the two-dimensional dilaton gravity [1-15] (see also [16-20] and references therein).

The Schwarzian theory inherits the symmetry properties that ensue from the time reparametrization independence of the physical picture. The point is that in some approximations, the above-mentioned physical models appear to be reparametrization invariant. However, in these models, this emergent reparametrization symmetry is broken to its $\mathrm{SL}(2, \mathbf{R})$ subgroup leading to the action

$$
-\int\left[\left(\frac{f^{\prime \prime}(t)}{f^{\prime}(t)}\right)^{\prime}-\frac{1}{2}\left(\frac{f^{\prime \prime}(t)}{f^{\prime}(t)}\right)^{2}\right] d t,
$$

which is the unique lowest order in derivatives action that is $\mathrm{SL}(2, \mathbf{R})$ invariant.

An extraordinary universality of the Schwarzian theory is a consequence of its rich symmetry structure. At the same time, one can use the invariance of the Schwarzian theory to link it to another theory [21] where the corresponding calculations are much simpler than in the original one (see also another approach in [22]). 
An idea of a slightly different way of studying the theory is to substitute the action

$$
\frac{1}{2} \int\left(\frac{f^{\prime \prime}(t)}{f^{\prime}(t)}\right)^{2} d t
$$

for the action (1.1). Now the correlation functions in SYK model can be written as the functional integrals of Liouville quantum mechanics. Using spectral decomposition, one can represent the functional integrals as the sums over the quantum eigenstates. Liouville theory is well studied, and the approach turned out to be very popular and fruitful [23-26].

Note however that the Lagrangian in (1.2) differs from that in (1.1) by a total derivative, and is not invariant under $\operatorname{SL}(2, \mathbf{R})$. The action $(1.2)$ is $\operatorname{SL}(2, \mathbf{R})$ invariant only if the boundary terms in the integral of the total derivative are equal to zero. In this case, Liouville quantum mechanics is an adequate way to handle the Schwarzian theory.

In general, a special technique of functional integration is needed in the Schwarzian theory. The point is that one should integrate over the elements of the group of diffeomorphisms and factor the (infinite) input of the $\mathrm{SL}(2, \mathbf{R})$ subgroup out. In this paper, we present such a technique and evaluate the functional integrals for correlation functions.

It is convenient to rewrite the action of the Schwarzian theory in the form

$$
I=-\frac{1}{\sigma^{2}} \int_{0}^{1}\left[\mathcal{S}_{\varphi}(t)+2 \pi^{2}\left(\varphi^{\prime}(t)\right)^{2}\right] d t
$$

where $\varphi(t)$ is a diffeomorphism of the interval $[0,1], f=\cot \pi \varphi$, and

$$
\mathcal{S}_{\varphi}(t)=\left(\frac{\varphi^{\prime \prime}(t)}{\varphi^{\prime}(t)}\right)^{\prime}-\frac{1}{2}\left(\frac{\varphi^{\prime \prime}(t)}{\varphi^{\prime}(t)}\right)^{2}
$$

is the Schwarzian derivative.

The presence of the term $\left(\varphi^{\prime}(t)\right)^{2}$ in the action (1.3) makes the functional integral

$$
\int F(\varphi) \exp \{-I\} d \varphi=\int F(\varphi) \exp \left\{\frac{1}{\sigma^{2}} \int_{0}^{1}\left[\mathcal{S}_{\varphi}(t)+2 \pi^{2}\left(\varphi^{\prime}(t)\right)^{2}\right] d t\right\} d \varphi
$$

look as if it is the integral over the Wiener measure

$$
w_{\kappa}(d \varphi)=\exp \left\{-\frac{1}{\kappa^{2}} \int_{0}^{1}\left(\varphi^{\prime}(t)\right)^{2} d t\right\} d \varphi
$$

analytically continued to the point $\kappa=i \frac{\sigma}{\sqrt{2} \pi}$. However, the attempt to treat (1.5) as the integral over the Wiener measure is a misleading one. The point is that the Wiener measure is concentrated on the trajectories that are nondifferentiable almost everywhere. The set of smooth, or even differentiable at a point, functions has zero Wiener measure. Nevertheless, the formal representation (1.6) is correct and very useful if the derivative $\varphi^{\prime}(t)$ is considered in a generalized sense [27]. However, the Schwarzian derivative cannot be understood in this way. 
A correct approach is based on the quasi-invariant measure on the group of diffeomorphisms (see the next section)

$$
\mu_{\sigma}(d \varphi)=\exp \left\{\frac{1}{\sigma^{2}} \int_{0}^{1} \mathcal{S}_{\varphi}(t) d t\right\} d \varphi
$$

And the functional integrals in the Schwarzian theory should be considered as the integrals over the measure (1.7).

In [28], the integral for the partition function in the Schwarzian theory

$$
Z_{\mathrm{Schw}}(g)=\frac{1}{\sqrt{2 \pi} g} \int_{\operatorname{Diff}^{1}\left(S^{1}\right) / \mathrm{SL}(2, \mathbf{R})} \exp \left\{\frac{1}{2 \pi g^{2}} \int_{0}^{1}\left[\mathcal{S}_{\varphi}(t)+2 \pi^{2}\left(\varphi^{\prime}(t)\right)^{2}\right] d t\right\} d \varphi
$$

was explicitly evaluated with the result

$$
Z_{\mathrm{Schw}}(g)=\frac{1}{2 \pi g^{3}} \exp \left\{\frac{\pi}{g^{2}}\right\}
$$

thereby confirming the conjecture about the exactness of the one-loop result [3, 21].

In this paper, we evaluate the functional integrals assigning correlation functions in the Schwarzian theory. As the technique of functional integration over quasi-invariant measures on infinite-dimensional groups is not common knowledge, we try to make the presentation maximally explicit giving the detailed proof of the basic formulas, and thus providing guidelines on the evaluation of functional integrals in the Schwarzian theory.

Section 2, and appendix A contain some preliminary material on quasi-invariant measures. In section 3, the explicit evaluation of the correlation functions is presented. In addition, some relevant technical results are obtained in appendices B, C and D. In section 4, we give the concluding remarks.

\section{Preliminaries}

For finite-dimensional groups, there is the invariant Haar measure. However, the invariant measures analogous to the Haar measure do not exist for the infinite-dimensional groups $H$ [29]. Nevertheless, sometimes one has succeeded in constructing the measure that is quasi-invariant with respect to the action of a more smooth subgroup $G \subset H$. The quasiinvariance means that under the action of the subgroup $G$ the measure transforms to itself multiplied by a function $\mathcal{R}_{g}(h)$ parametrized by the elements of the subgroup $g \in G$

$$
\mu(d(g \circ h))=\mathcal{R}_{g}^{\mu}(h) \mu(d h) .
$$

The function $\mathcal{R}_{g}^{\mu}(h)$ is called the Radon-Nikodim derivative of the measure $\mu$ (see, e.g., $[27,30])$.

The quasi-invariance of the Wiener measure (1.6) under the shifts of the argument of the measure by a differentiable function is the simplest example [27]. 
The Wiener measure (1.6) turns out to be quasi-invariant under the group of diffeomorphisms. The proof of the quasi-invariance and the explicit form of the Radon-Nikodim derivative was first obtained in [31] (see a more simple derivation of the result, e.g., in [32]).

The evaluation of the functional integrals considered in this paper is based on the equation:

$$
\int_{\xi(0)=\xi(1)=0} \exp \left\{\frac{-2 \beta^{2}}{\sigma^{2}(\beta+1)} \frac{1}{\int_{0}^{1} e^{\xi(t)} d t}\right\} w_{\sigma}(d \xi)=\frac{1}{\sqrt{2 \pi} \sigma} \exp \left\{-\frac{2}{\sigma^{2}}(\log (\beta+1))^{2}\right\} .
$$

It is a consequence of the quasi-invariance of the Wiener measure with respect to the action of the group of diffeomorphisms $\operatorname{Diff}_{+}^{3}([0,1])$. In [28], we postponed the proof of the equation (2.1) till the next paper, "Hanc marginis exiguitas non caparet." (P. Fermat).

Now, the explicit proof of the more general formula

$$
\begin{array}{r}
\int_{\xi(0)=0, \xi(1)=x} \exp \left\{\frac{-2 \beta}{\sigma^{2}}\left[1-\frac{e^{x}}{\beta+1}\right] \frac{1}{\int_{0}^{1} e^{\xi(t)} d t}\right\} w_{\sigma}(d \xi) \\
=\frac{1}{\sqrt{2 \pi} \sigma} \exp \left\{-\frac{1}{2 \sigma^{2}}(x-2 \log (\beta+1))^{2}\right\}
\end{array}
$$

is given in [33] (see, also, appendix B of the present paper).

In [34-36], the measures

$$
\mu_{\sigma}(X)=\int_{X} \exp \left\{\frac{1}{\sigma^{2}} \int_{0}^{1} \mathcal{S}_{\varphi}(t) d t\right\} d \varphi
$$

on the groups of diffeomorphisms of the interval $X \subset \operatorname{Diff}_{+}^{1}([0,1])$, and of the circle $X \subset$ $\operatorname{Diff}_{+}^{1}\left(S^{1}\right)$ were proposed.

The measures are quasi-invariant with respect to the action of the subgroups $\operatorname{Diff}_{+}^{3}([0,1])$ and $\operatorname{Diff}_{+}^{3}\left(S^{1}\right)$ respectively. The proof of the quasi-invariance and the form of the Radon-Nikodim derivatives can be obtained by some special substitution of variables [34-36] (see, also, appendix A of the present paper). Specifically, under the substitution

$$
\varphi(t)=\frac{\int_{0}^{t} e^{\xi(\tau)} d \tau}{\int_{0}^{1} e^{\xi(\eta)} d \eta}
$$

the measure $\mu_{\sigma}(d \varphi)$ on the group $\operatorname{Diff}_{+}^{1}([0,1])$ turns into the Wiener measure $w_{\sigma}(d \xi)$ on $C([0,1])$.

Consider the function $\xi \in C_{0}([0,1])$. That is, $\xi(t)$ is a continuous function on the interval satisfying the boundary condition $\xi(0)=0$. Then

$$
\xi(t)=\log \varphi^{\prime}(t)-\log \varphi^{\prime}(0) .
$$


The integral over the group $\operatorname{Diff}^{1}\left(S^{1}\right)$ can be transformed into the integral over the group $\operatorname{Diff}^{1}([0,1])$. Note that if we fix a point $t=0$ on the circle $S^{1}$ then it is necessary "to glue the ends of the interval". That is, to put $\varphi^{\prime}(0)=\varphi^{\prime}(1)$ or $\xi(0)=\xi(1)=0$. In this case, the function $\xi$ is a Brownian bridge, and we denote the corresponding functional space by $C_{0,0}([0,1])$. The Wiener measures on $C_{0}([0,1])$ and $C_{0,0}([0,1])$ are related by the equation

$$
w_{\sigma}(d \xi)=w_{\sigma}^{\text {Brown }}(d \xi) \frac{1}{\sqrt{2 \pi} \sigma} \exp \left(-\frac{x^{2}}{2 \sigma^{2}}\right) d x
$$

Now, the integral over $\operatorname{Diff}^{1}\left(S^{1}\right)$ turns into the integral over $\operatorname{Diff}^{1}([0,1])$ as follows:

$$
\begin{aligned}
& \frac{1}{\sqrt{2 \pi} \sigma} \int_{\operatorname{Diff}^{1}\left(S^{1}\right)} F(\varphi) \mu_{\sigma}(d \varphi) \\
& =\frac{1}{\sqrt{2 \pi} \sigma} \int_{C_{0,0}([0,1])} F(\varphi(\xi)) w_{\sigma}^{\text {Brown }}(d \xi)=\int_{C_{0}([0,1])} \delta(\xi(1)) F(\varphi(\xi)) w_{\sigma}(d \xi) \\
& =\int_{\operatorname{Diff}^{1}([0,1])} \delta\left(\log \frac{\varphi^{\prime}(1)}{\varphi^{\prime}(0)}\right) F(\varphi) \mu_{\sigma}(d \varphi)=\int_{\operatorname{Diff}^{1}([0,1])} \delta\left(\frac{\varphi^{\prime}(1)}{\varphi^{\prime}(0)}-1\right) F(\varphi) \mu_{\sigma}(d \varphi) .
\end{aligned}
$$

Here, we have used the equation

$$
\int_{0}^{+\infty} \delta(\log x) g(x) d x=g(1)=\int_{-\infty}^{+\infty} \delta(x-1) g(x) d x .
$$

The quasi-invariance of the measure and the explicit form of the Radon-Nikodim derivative can be used to evaluate nontrivial functional integrals. For the measure $\mu$ on the interval $[0,1]$ in particular, the Radon-Nikodim derivative is

$$
\begin{aligned}
\mathcal{R}_{f}^{\mu}(\varphi) \equiv \frac{d \mu_{\sigma}^{f}}{d \mu_{\sigma}}(\varphi)= & \frac{1}{\sqrt{f^{\prime}(0) f^{\prime}(1)}} \\
& \times \exp \left\{\frac{1}{\sigma^{2}}\left[\frac{f^{\prime \prime}(0)}{f^{\prime}(0)} \varphi^{\prime}(0)-\frac{f^{\prime \prime}(1)}{f^{\prime}(1)} \varphi^{\prime}(1)\right]+\frac{1}{\sigma^{2}} \int_{0}^{1} \mathcal{S}_{f}(\varphi(t))\left(\varphi^{\prime}(t)\right)^{2} d t\right\},
\end{aligned}
$$

where

$$
\mu_{\sigma}^{f}(X)=\mu_{\sigma}(f \circ X) .
$$

Here, the well known property of the Schwarzian derivative:

$$
\mathcal{S}_{f \circ \varphi}(t)=\mathcal{S}_{f}(\varphi(t))\left(\varphi^{\prime}(t)\right)^{2}+\mathcal{S}_{\varphi}(t), \quad(f \circ \varphi)(t)=f(\varphi(t)),
$$

has been used.

Thus, for functional integrals over the measure $\mu$, we have

$$
\begin{aligned}
& \int_{\operatorname{Diff}^{1}([0,1])} F(\varphi) \mu_{\sigma}(d \varphi)=\frac{1}{\sqrt{f^{\prime}(0) f^{\prime}(1)}} \int_{\operatorname{Diff}^{1}([0,1])} F(f \circ \varphi) \\
& \quad \times \exp \left\{\frac{1}{\sigma^{2}}\left[\frac{f^{\prime \prime}(0)}{f^{\prime}(0)} \varphi^{\prime}(0)-\frac{f^{\prime \prime}(1)}{f^{\prime}(1)} \varphi^{\prime}(1)\right]+\frac{1}{\sigma^{2}} \int_{0}^{1} \mathcal{S}_{f}(\varphi(t))\left(\varphi^{\prime}(t)\right)^{2} d t\right\} \mu_{\sigma}(d \varphi) .
\end{aligned}
$$


In what follows, we assume the function $f$ to be

$$
f(t)=f_{\alpha}(t)=\frac{1}{2}\left[\frac{1}{\tan \frac{\alpha}{2}} \tan \left(\alpha\left(t-\frac{1}{2}\right)\right)+1\right] .
$$

In this case,

$$
f_{\alpha}^{\prime}(0)=f_{\alpha}^{\prime}(1)=\frac{\alpha}{\sin \alpha}, \quad-\frac{f_{\alpha}^{\prime \prime}(0)}{f_{\alpha}^{\prime}(0)}=\frac{f_{\alpha}^{\prime \prime}(1)}{f_{\alpha}^{\prime}(1)}=2 \alpha \tan \frac{\alpha}{2}, \quad \mathcal{S}_{f_{\alpha}}(t)=2 \alpha^{2},
$$

and the equation (2.8) looks like:

$$
\begin{aligned}
& \frac{\alpha}{\sin \alpha} \int_{\operatorname{Diff}^{1}([0,1])} F(\varphi) \mu_{\sigma}(d \varphi)=\int_{\operatorname{Diff}^{1}([0,1])} F\left(f_{\alpha}(\varphi)\right) \\
& \quad \times \exp \left\{-\frac{2 \alpha}{\sigma^{2}} \tan \frac{\alpha}{2}\left(\varphi^{\prime}(0)+\varphi^{\prime}(1)\right)\right\} \exp \left\{\frac{2 \alpha^{2}}{\sigma^{2}} \int_{0}^{1}\left(\varphi^{\prime}(t)\right)^{2} d t\right\} \mu_{\sigma}(d \varphi) .
\end{aligned}
$$

Generally speaking, the functional integrals (2.11) converge for $0 \leq \alpha<\pi$, and diverge for $\alpha=\pi$.

The Schwarzian action is invariant under the noncompact group $\operatorname{SL}(2, \mathbf{R})$. Therefore, integrating over the quotient space $\operatorname{Diff}^{1}([0,1]) / \operatorname{SL}(2, \mathbf{R})$ we exclude the infinite volume of the group $\operatorname{SL}(2, \mathbf{R})$ and get the finite results for functional integrals in the Schwarzian theory. In our approach, we evaluate regularized $(\alpha<\pi)$ functional integrals over the group $\operatorname{Diff}^{1}([0,1])$ and then normalize them to the corresponding integrals over the group $\operatorname{SL}(2, \mathbf{R})$.

In particular, in [28], to get the partition function (1.9), we first evaluated the regularized integral

$$
\begin{aligned}
Z_{\alpha}(\sigma) & =\frac{1}{\sqrt{2 \pi} \sigma} \int_{\operatorname{Diff}^{1}\left(S^{1}\right)} \exp \{-I\} \exp \left\{\frac{-2\left[\pi^{2}-\alpha^{2}\right]}{\sigma^{2}} \int_{0}^{1}\left(\varphi^{\prime}(t)\right)^{2} d t\right\} d \varphi \\
& =\frac{1}{\sqrt{2 \pi} \sigma} \int_{\operatorname{Diff}^{1}\left(S^{1}\right)} \exp \left\{\frac{2 \alpha^{2}}{\sigma^{2}} \int_{0}^{1}\left(\varphi^{\prime}(t)\right)^{2} d t\right\} \mu_{\sigma}(d \varphi),
\end{aligned}
$$

and then divided it by the regularized volume of the group $\mathrm{SL}(2, \mathbf{R})$

$$
V_{\alpha}(\sigma)=\int_{\operatorname{SL}(2, \mathbf{R})} \exp \left\{\frac{-2\left[\pi^{2}-\alpha^{2}\right]}{\sigma^{2}} \int_{0}^{1}\left(\varphi^{\prime}(t)\right)^{2} d t\right\} d \nu .
$$

Note that the functional measure in the equation (2.12) and the Haar measure $d \nu$ on the group $\operatorname{SL}(2, \mathbf{R})$ in the equation (2.13) are regularized in the same manner.

For the Schwarzian partition function, we take the limit

$$
Z(\sigma)=\lim _{\alpha \rightarrow \pi-0} \frac{Z_{\alpha}(\sigma)}{V_{\alpha}(\sigma)} .
$$

In the next section, the quasi-invariance of the measure (1.7) is used to evaluate the functional integrals assigning the correlation functions in the Schwarzian theory. 


\section{Correlation functions}

\subsection{Mean value of $\varphi^{\prime}$}

First we recall the main steps of the evaluation of the partition function in the Schwarzian theory [28].

If we take the function $F$ in the equation (2.11) to be

$$
F(f(\varphi))=F_{1}(f(\varphi))=\exp \left\{\frac{4 \alpha}{\sigma^{2}} \tan \frac{\alpha}{2} \varphi^{\prime}(0)\right\},
$$

and note that

$$
\varphi^{\prime}(0)=\frac{1}{f^{\prime}(0)} u^{\prime}(0)=\frac{\sin \alpha}{\alpha} u^{\prime}(0),
$$

for $u(t)=f(\varphi(t))$, then

$$
F_{1}(u)=\exp \left\{\frac{8 \sin ^{2} \frac{\alpha}{2}}{\sigma^{2}} u^{\prime}(0)\right\} .
$$

Now the regularized partition function has the form

$$
Z_{\alpha}(\sigma)=\frac{\alpha}{\sin \alpha} \frac{1}{\sqrt{2 \pi} \sigma} \int_{\operatorname{Diff}^{1}\left(S^{1}\right)} \exp \left\{\frac{8 \sin ^{2} \frac{\alpha}{2}}{\sigma^{2}} \varphi^{\prime}(0)\right\} \mu_{\sigma}(d \varphi) .
$$

Under the substitution (2.4) it turns into

$$
Z_{\alpha}(\sigma)=\frac{\alpha}{\sin \alpha} \frac{1}{\sqrt{2 \pi} \sigma} \int_{\operatorname{Diff}^{1}\left(S^{1}\right)} \exp \left\{\frac{8 \sin ^{2} \frac{\alpha}{2}}{\sigma^{2}} \frac{1}{\int_{0}^{1} e^{\xi(\tau)} d \tau}\right\} w_{\sigma}(d \xi) .
$$

To evaluate the functional integral explicitly we use the equation (2.1). Instead of $\beta$, we should substitute a solution of the equation

$$
\frac{2 \beta^{2}}{\sigma^{2}(\beta+1)}=-\frac{8 \sin ^{2} \frac{\alpha}{2}}{\sigma^{2}} .
$$

We take the following one:

$$
(\beta+1)=e^{i \alpha}
$$

As the result, we obtain

$$
Z_{\alpha}(\sigma)=\frac{\alpha}{\sin \alpha} \frac{1}{\sqrt{2 \pi} \sigma} \exp \left\{\frac{2 \alpha^{2}}{\sigma^{2}}\right\}
$$

with the asymptotic form at $\alpha \rightarrow \pi$

$$
Z_{\alpha}^{A s}(\sigma)=\frac{\pi}{\pi-\alpha} \frac{1}{\sqrt{2 \pi} \sigma} \exp \left\{\frac{2 \pi^{2}}{\sigma^{2}}\right\} .
$$


The asymptotic form of the $\alpha$-regularized volume of the group $\operatorname{SL}(2, \mathbf{R}$ ) (see appendix D) looks like

$$
V_{\alpha ; \mathrm{SL}(2, \mathbf{R})}^{A s}(\sigma)=\frac{\sigma^{2}}{2[\pi-\alpha]}
$$

According to the equation (2.14), the Schwarzian partition function has the form

$$
Z(\sigma)=\frac{\sqrt{2 \pi}}{\sigma^{3}} \exp \left\{\frac{2 \pi^{2}}{\sigma^{2}}\right\}
$$

Consider now the $\alpha$-regularized mean value of $\varphi^{\prime}$

$$
\Phi^{\alpha}=\frac{1}{\sqrt{2 \pi} \sigma} \int_{\operatorname{Diff}^{1}\left(S^{1}\right)} \varphi^{\prime}(0) \exp \left\{\frac{2 \alpha^{2}}{\sigma^{2}} \int_{0}^{1}\left(\varphi^{\prime}(\tau)\right)^{2} d \tau\right\} \mu_{\sigma}(d \varphi)
$$

Note that it is $\varphi^{\prime}$, but not $\varphi$, that is the dynamical variable in the theory given by the action (1.3).

After the substitution (2.4), it is written as

$$
\Phi^{\alpha}=\frac{1}{\sqrt{2 \pi} \sigma} \int_{\operatorname{Diff}^{1}\left(S^{1}\right)} \frac{1}{\int_{0}^{1} e^{\xi(\tau)} d \tau} \exp \left\{\frac{8 \sin ^{2} \frac{\alpha}{2}}{\sigma^{2}} \frac{1}{\int_{0}^{1} e^{\xi(\tau)} d \tau}\right\} w_{\sigma}(d \xi)
$$

Having in mind the equations (2.1), (3.5), and (3.6), we get

$$
\Phi^{\alpha}=\frac{4}{\sqrt{2 \pi} \sigma^{3}} \frac{\ln (\beta+1)}{(\beta+1)} \exp \left\{-\frac{2 \ln ^{2}(\beta+1)}{\sigma^{2}}\right\}=\frac{\alpha}{\sin \alpha} \frac{1}{\sqrt{2 \pi} \sigma} \exp \left\{\frac{2 \alpha^{2}}{\sigma^{2}}\right\} .
$$

Asymptotically, it looks like

$$
\Phi^{A s}=\frac{\pi}{\pi-\alpha} \frac{1}{\sqrt{2 \pi} \sigma} \exp \left\{\frac{2 \pi^{2}}{\sigma^{2}}\right\}
$$

The asymptotic form of the $\Phi^{\alpha}$ on the group $\operatorname{SL}(2, \mathbf{R})$ (see appendix D) is

$$
\Phi_{\mathrm{SL}(2, \mathbf{R})}^{A s}=\frac{\sigma^{2}}{2[\pi-\alpha]} .
$$

Now the normalized mean value of $\varphi^{\prime}$ has the form

$$
\Phi=\lim _{\alpha \rightarrow \pi-0} \frac{\Phi^{A s}}{\Phi_{\mathrm{SL}(2, \mathbf{R})}^{A s}}=\frac{\sqrt{2 \pi}}{\sigma^{3}} \exp \left\{\frac{2 \pi^{2}}{\sigma^{2}}\right\}
$$




\subsection{Two-point correlation function}

Define the $\alpha$-regularized two-point correlation function as

$$
\begin{aligned}
G_{2}^{\alpha}(0, t) & =\frac{1}{\sqrt{2 \pi} \sigma} \int_{\operatorname{Diff}^{1}\left(S^{1}\right)} \varphi^{\prime}(t) \varphi^{\prime}(0) \exp \left\{\frac{2 \alpha^{2}}{\sigma^{2}} \int_{0}^{1}\left(\varphi^{\prime}(\tau)\right)^{2} d \tau\right\} \mu_{\sigma}(d \varphi) \\
& =\int_{\operatorname{Diff}^{1}([0,1])} \varphi^{\prime}(t) \varphi^{\prime}(0) \delta\left(\frac{\varphi^{\prime}(1)}{\varphi^{\prime}(0)}-1\right) \exp \left\{\frac{2 \alpha^{2}}{\sigma^{2}} \int_{0}^{1}\left(\varphi^{\prime}(\tau)\right)^{2} d \tau\right\} \mu_{\sigma}(d \varphi) .
\end{aligned}
$$

By the special choice of the function $F$ in (2.11), we identify the integrands in (3.16) and in the right-hand side of the equation (2.11).

Represent the function $F$ in the form

$$
F(\varphi)=F_{4}(\varphi) F_{3}(\varphi) F_{2}(\varphi) F_{1}(\varphi)
$$

where

$$
\begin{array}{ll}
F_{1}(f \circ \varphi)=\exp \left\{\frac{4 \alpha}{\sigma^{2}} \tan \frac{\alpha}{2} \varphi^{\prime}(0)\right\}, & F_{2}(f \circ \varphi)=\delta\left(\varphi^{\prime}(1)-\varphi^{\prime}(0)\right), \\
F_{3}(f \circ \varphi)=\left(\varphi^{\prime}(0)\right)^{2}, & F_{4}(f \circ \varphi)=\varphi^{\prime}(t) .
\end{array}
$$

To use the equation $(2.11)$, it is necessary to find $F_{i}(\varphi) . F_{1}(\varphi)$ was found above to be

$$
F_{1}(\varphi)=\exp \left\{\frac{8 \sin ^{2} \frac{\alpha}{2}}{\sigma^{2}} \varphi^{\prime}(0)\right\} \text {. }
$$

To find $F_{2}(\varphi)$ and $F_{3}(\varphi)$, note that for $\chi(t)=f(\varphi(t))$,

$$
\chi^{\prime}(0)=\frac{\alpha}{\sin \alpha} \varphi^{\prime}(0), \quad \chi^{\prime}(1)=\frac{\alpha}{\sin \alpha} \varphi^{\prime}(1),
$$

so

$$
F_{2}(\varphi)=\frac{\alpha}{\sin \alpha} \delta\left(\varphi^{\prime}(1)-\varphi^{\prime}(0)\right)
$$

and

$$
F_{3}(\varphi)=\frac{\sin ^{2} \alpha}{\alpha^{2}}\left(\varphi^{\prime}(0)\right)^{2}
$$

$F_{4}(\varphi)$ looks more complicated:

$$
F_{4}(\chi(t))=F_{4}(f(\varphi(t)))=\varphi^{\prime}(t)=\frac{1}{f^{\prime}\left(f^{-1}(\chi(t))\right)} \chi^{\prime}(t)=\left(f^{-1}\right)^{\prime}(\chi(t)) \chi^{\prime}(t) .
$$

For the function $y=f_{\alpha}(x)$ given by the equation (2.9),

$$
x=f_{\alpha}^{-1}(y)=\frac{1}{\alpha} \arctan \left[\tan \frac{\alpha}{2}(2 y-1)\right]+\frac{1}{2} .
$$

And finally,

$$
F_{4}(\varphi)=\frac{\frac{2}{\alpha} \tan \frac{\alpha}{2}}{1+\tan ^{2} \frac{\alpha}{2}(2 \varphi(t)-1)^{2}} \varphi^{\prime}(t) .
$$


Thus for the correlation function, we have

$$
\begin{aligned}
G_{2}^{\alpha}(0, t)= & \int_{\operatorname{Diff}^{1}([0,1])} \frac{\frac{2}{\alpha} \tan \frac{\alpha}{2}}{1+\tan ^{2} \frac{\alpha}{2}(2 \varphi(t)-1)^{2}} \\
& \times \varphi^{\prime}(t) \varphi^{\prime}(0) \delta\left(\frac{\varphi^{\prime}(1)}{\varphi^{\prime}(0)}-1\right) \exp \left\{\frac{8 \sin ^{2} \frac{\alpha}{2}}{\sigma^{2}} \varphi^{\prime}(0)\right\} \mu_{\sigma}(d \varphi) \\
= & \int_{0}^{1} \frac{\frac{2}{\alpha} \tan \frac{\alpha}{2}}{1+\tan ^{2} \frac{\alpha}{2}(2 x-1)^{2}} d x \int_{\operatorname{Diff}^{1}([0,1])} \delta(x-\varphi(t)) \\
& \times \varphi^{\prime}(t) \varphi^{\prime}(0) \delta\left(\frac{\varphi^{\prime}(1)}{\varphi^{\prime}(0)}-1\right) \exp \left\{\frac{8 \sin ^{2} \frac{\alpha}{2}}{\sigma^{2}} \varphi^{\prime}(0)\right\} \mu_{\sigma}(d \varphi) .
\end{aligned}
$$

The substitution (2.4) in the above equation gives the correlation function in terms of the Wiener integral:

$$
\begin{aligned}
G_{2}^{\alpha}(0, t)= & \int_{0}^{1} \frac{\frac{2}{\alpha} \tan \frac{\alpha}{2}}{1+\tan ^{2} \frac{\alpha}{2}(2 x-1)^{2}} d x \int_{C_{0}([0,1])} \delta\left(x \int_{0}^{1} e^{\xi(\tau)} d \tau-\int_{0}^{t} e^{\xi(\tau)} d \tau\right) \\
& \times \frac{e^{\xi(t)}}{\int_{0}^{1} e^{\xi(\tau)} d \tau} \delta\left(e^{\xi(1)}-1\right) \exp \left\{\frac{8 \sin ^{2} \frac{\alpha}{2}}{\sigma^{2}} \frac{1}{\int_{0}^{1} e^{\xi(\tau)} d \tau}\right\} w_{\sigma}(d \xi) .
\end{aligned}
$$

Divide the interval $[0,1]$ into the two intervals $[0, t]$ and $[t, 1]$. The substitution

$$
\xi(\tau)=\eta_{1}\left(\frac{\tau}{t}\right), \quad \tau \leq t ; \quad \xi(\tau)=\eta_{1}(1)+\eta_{2}\left(\frac{\tau-t}{1-t}\right), \quad \tau>t
$$

transforms the Wiener integral over the measure $w_{\sigma}(d \xi)$ into the two Wiener integrals over the measures

$$
w_{\sigma \sqrt{t}}\left(d \eta_{1}\right) w_{\sigma \sqrt{1-t}}\left(d \eta_{2}\right) .
$$

To verify this statement, note that

$$
\int_{0}^{t}\left(\xi^{\prime}(\tau)\right)^{2} d \tau=\frac{1}{t} \int_{0}^{1}\left(\eta_{1}^{\prime}(\tau)\right)^{2} d \tau, \quad \int_{t}^{1}\left(\xi^{\prime}(\tau)\right)^{2} d \tau=\frac{1}{1-t} \int_{0}^{1}\left(\eta_{2}^{\prime}(\tau)\right)^{2} d \tau .
$$

To return to the integrals over the group of diffeomorphisms, consider the functions

$$
\psi_{1}(t)=\frac{\int_{0}^{t} e^{\eta_{1}(\tau)} d \tau}{\int_{0}^{1} e^{\eta_{1}(\tau)} d \tau}, \quad \psi_{2}(t)=\frac{\int_{0}^{t} e^{\eta_{2}(\tau)} d \tau}{\int_{0}^{1} e^{\eta_{2}(\tau)} d \tau} .
$$


The useful relations can be obtained directly from the above definitions

$$
\begin{aligned}
\varphi(t) & =\frac{t \psi_{2}^{\prime}(0)}{t \psi_{2}^{\prime}(0)+(1-t) \psi_{1}^{\prime}(1)}, & \varphi^{\prime}(t) & =\frac{\psi_{1}^{\prime}(1) \psi_{2}^{\prime}(0)}{t \psi_{2}^{\prime}(0)+(1-t) \psi_{1}^{\prime}(1)}, \\
\varphi^{\prime}(0) & =\frac{\psi_{1}^{\prime}(0) \psi_{2}^{\prime}(0)}{t \psi_{2}^{\prime}(0)+(1-t) \psi_{1}^{\prime}(1)}, & \varphi^{\prime}(1) & =\frac{\psi_{1}^{\prime}(1) \psi_{2}^{\prime}(1)}{t \psi_{2}^{\prime}(0)+(1-t) \psi_{1}^{\prime}(1)} .
\end{aligned}
$$

Therefore, the two-point correlation function has the form of the double functional integral

$$
\begin{aligned}
G_{2}^{\alpha}(0, t)= & \frac{1}{t(1-t)} \int_{0}^{1} \frac{\frac{2}{\alpha} \tan \frac{\alpha}{2}}{1+\tan ^{2} \frac{\alpha}{2}(2 x-1)^{2}} d x \int_{\operatorname{Diff}^{1}([0,1])} \int_{\operatorname{Diff}^{1}([0,1])}\left(\psi_{1}^{\prime}(1)\right)^{2}\left(\psi_{2}^{\prime}(1)\right)^{2} \\
& \times \delta\left(\psi_{2}^{\prime}(0)-\frac{(1-t)}{t} \frac{x}{1-x} \psi_{1}^{\prime}(1)\right) \delta\left(\psi_{1}^{\prime}(0)-\frac{t}{1-t} \frac{1-x}{x} \psi_{2}^{\prime}(1)\right) \\
& \times \exp \left\{\frac{8 \sin ^{2} \frac{\alpha}{2}}{\sigma^{2}} \frac{(1-x)}{(1-t)} \psi_{2}^{\prime}(1)\right\} \mu_{\sigma \sqrt{t}}\left(d \psi_{1}\right) \mu_{\sigma \sqrt{1-t}}\left(d \psi_{2}\right)
\end{aligned}
$$

Define the function $\mathcal{E}_{\sigma}(u, v)$ by the equation

$$
\mathcal{E}_{\sigma}(u, v)=\int_{\operatorname{Diff}^{1}([0,1])} \delta\left(\varphi^{\prime}(0)-u\right) \delta\left(\varphi^{\prime}(1)-v\right) \mu_{\sigma}(d \varphi)
$$

We can rewrite the equation (3.25) as

$$
\begin{aligned}
G_{2}^{\alpha}(0, t)= & \frac{1}{t(1-t)} \int_{0}^{1} \frac{\frac{2}{\alpha} \tan \frac{\alpha}{2}}{1+\tan ^{2} \frac{\alpha}{2}(2 x-1)^{2}} d x \int_{0}^{+\infty} \int_{0}^{+\infty} v_{1}^{2} v_{2}^{2} \exp \left\{\frac{8 \sin ^{2} \frac{\alpha}{2}}{\sigma^{2}} \frac{(1-x)}{(1-t)} v_{2}\right\} \\
& \times \mathcal{E}_{\sigma \sqrt{t}}\left(\frac{t}{1-t} \frac{1-x}{x} v_{2}, v_{1}\right) \mathcal{E}_{\sigma \sqrt{1-t}}\left(\frac{1-t}{t} \frac{x}{1-x} v_{1}, v_{2}\right) d v_{1} d v_{2} .
\end{aligned}
$$

In terms of variables

$$
V_{1}=\frac{x}{t} v_{1}, \quad V_{2}=\frac{1-x}{1-t} v_{2},
$$

the correlation function looks like

$$
\begin{aligned}
G_{2}^{\alpha}(0, t)= & t^{2}(1-t)^{2} \int_{0}^{1} \frac{1}{x^{3}(1-x)^{3}} \frac{\frac{2}{\alpha} \tan \frac{\alpha}{2}}{1+\tan ^{2} \frac{\alpha}{2}(2 x-1)^{2}} d x \int_{0}^{+\infty} \int_{0}^{+\infty} V_{1}^{2} V_{2}^{2} \\
& \times \exp \left\{\frac{8 \sin ^{2} \frac{\alpha}{2}}{\sigma^{2}} V_{2}\right\} \mathcal{E}_{\sigma \sqrt{t}}\left(\frac{t}{x} V_{2}, \frac{t}{x} V_{1}\right) \mathcal{E}_{\sigma \sqrt{1-t}}\left(\frac{1-t}{1-x} V_{1}, \frac{1-t}{1-x} V_{2}\right) d V_{1} d V_{2} .
\end{aligned}
$$


In appendix $\mathrm{C}$, we study the properties of the function $\mathcal{E}$ and, in particular, obtain the equations

$$
\begin{aligned}
\mathcal{E}_{\sigma}(u, v)= & \mathcal{E}_{\sigma}(\sqrt{u v}, \sqrt{u v}) \exp \left\{-\frac{2}{\sigma^{2}}(\sqrt{u}-\sqrt{v})^{2}\right\} \\
\mathcal{E}_{\sigma}(u, u)= & \frac{2 \sqrt{2}}{u \pi^{\frac{3}{2}} \sigma^{3}} \exp \left\{\frac{2 \pi^{2}-4 u}{\sigma^{2}}\right\} \\
& \times \int_{0}^{+\infty} \exp \left\{-\frac{2}{\sigma^{2}}\left(2 u \cosh \tau+\tau^{2}\right)\right\} \sin \left(\frac{4 \pi \tau}{\sigma^{2}}\right) \sinh (\tau) d \tau
\end{aligned}
$$

Now, using the equations (3.29), (3.30) and the substitution $z=(2 x-1) \tan \frac{\alpha}{2}$, we get the following representation for the correlation function in terms of the ordinary integrals:

$$
\begin{aligned}
G_{2}^{\alpha}(0, t)= & \frac{8^{3}}{\pi^{3} \sigma^{6}} \frac{1}{\sqrt{t(1-t)}} \exp \left\{\frac{2 \pi^{2}}{\sigma^{2} t(1-t)}\right\} \int_{0}^{+\infty} \int_{0}^{+\infty} H(\alpha ; \tau, \theta) \\
& \times \exp \left\{-\frac{2}{\sigma^{2}}\left[\frac{\tau^{2}}{t}+\frac{\theta^{2}}{(1-t)}\right]\right\} \sin \left(\frac{4 \pi \tau}{\sigma^{2} t}\right) \sin \left(\frac{4 \pi \theta}{\sigma^{2}(1-t)}\right) \sinh (\tau) \sinh (\theta) d \tau d \theta
\end{aligned}
$$

where

$$
\begin{aligned}
H(\alpha ; \tau, \theta)= & \frac{1}{\alpha} \int_{-\tan \frac{\alpha}{2}}^{+\tan \frac{\alpha}{2}} \frac{1}{\left[1-z^{2} \cot ^{2} \frac{\alpha}{2}\right]^{2}} \frac{d z}{1+z^{2}} \\
& \times \int_{0}^{+\infty} \int_{0}^{+\infty} d V_{1} d V_{2} V_{1} V_{2} \exp \left\{-\frac{8}{\sigma^{2}} \frac{1}{\left(1-z^{2} \cot ^{2} \frac{\alpha}{2}\right)}\left(\left(1+z^{2}\right) V_{2} \cos ^{2} \frac{\alpha}{2}+V_{1}\right)\right\} \\
& \times \exp \left\{-\frac{8}{\sigma^{2}}\left(\frac{\cosh (\tau)}{1+z \cot \frac{\alpha}{2}}+\frac{\cosh (\theta)}{1-z \cot \frac{\alpha}{2}}\right) \sqrt{V_{1} V_{2}}\right\}
\end{aligned}
$$

Note that the integrals in (3.32) are convergent because of the decreasing exponent in the integrand.

The generalized polar substitution

$$
V_{1}=\rho \sin ^{2} \omega, V_{2}=\rho \cos ^{2} \omega,
$$

and the substitution $y=\tan \omega$ reduce the equation (3.32) to the table integrals (see, e.g., [37] n. 2.2.9.11) with the result

$$
\begin{aligned}
H(\alpha ; \tau, \theta)= & 12\left(\frac{\sigma^{2}}{8}\right)^{4} \frac{1}{(\cosh (\tau)+\cosh (\theta))^{4}} \\
& \times\left\{-\log \sin \alpha+\log (\cosh \tau+\cosh \theta)-\frac{3}{2} \log 2+\log \sigma-\frac{11}{3}+O(\pi-\alpha)\right\}
\end{aligned}
$$




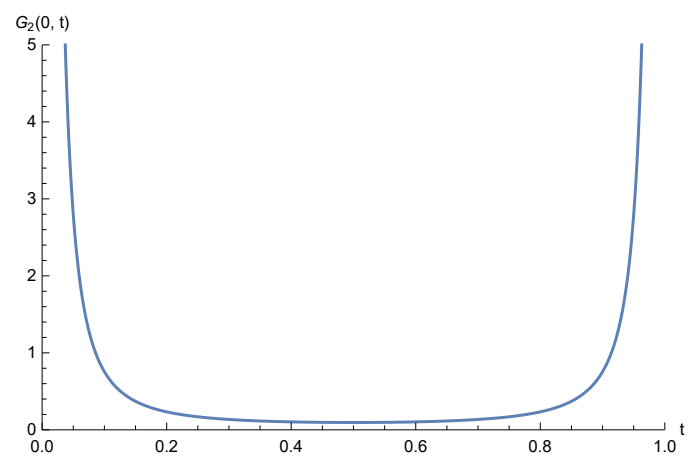

Figure 1. The form of the correlation function $G_{2}(0, t)$ for $\sigma=2 \pi$.

The asymptotics of the two-point correlation function at $\alpha \rightarrow \pi$ has the form

$$
\begin{aligned}
G_{2}^{A s}(0, t)= & \frac{3 \sigma^{2}}{2 \pi^{3}} \frac{(-\log \sin \alpha)}{\sqrt{t(1-t)}} \exp \left\{\frac{2 \pi^{2}}{\sigma^{2} t(1-t)}\right\} \int_{0}^{+\infty} \int_{0}^{+\infty} \exp \left\{-\frac{2}{\sigma^{2}}\left[\frac{\tau^{2}}{t}+\frac{\theta^{2}}{(1-t)}\right]\right\} \\
& \times \sin \left(\frac{4 \pi \tau}{\sigma^{2} t}\right) \sin \left(\frac{4 \pi \theta}{\sigma^{2}(1-t)}\right) \frac{\sinh (\tau) \sinh (\theta)}{(\cosh (\tau)+\cosh (\theta))^{4}} d \tau d \theta .
\end{aligned}
$$

Define the normalized two-point correlation function $G_{2}(0, t)$ as the limit

$$
G_{2}(0, t)=\lim _{\alpha \rightarrow \pi-0} \frac{G_{2}^{\alpha}(0, t)}{G_{2 ; \operatorname{SL}(2, \mathbf{R})}^{\alpha}\left(0, \frac{1}{2}\right)} .
$$

Here, the correlation function on the group $\operatorname{SL}(2, \mathbf{R})$ at the symmetrical points is chosen as the normalizing factor. It is evaluated in appendix D with the result

$$
G_{2 ; \mathrm{SL}(2, \mathbf{R})}^{A s}\left(0, \frac{1}{2}\right)=-\pi \log (\pi-\alpha) .
$$

Thus we have

$$
\begin{aligned}
G_{2}(0, t)= & \frac{3 \sigma^{2}}{2 \pi^{4}} \frac{1}{\sqrt{t(1-t)}} \exp \left\{\frac{2 \pi^{2}}{\sigma^{2} t(1-t)}\right\} \int_{0}^{+\infty} \int_{0}^{+\infty} \exp \left\{-\frac{2}{\sigma^{2}}\left[\frac{\tau^{2}}{t}+\frac{\theta^{2}}{(1-t)}\right]\right\} \\
& \times \sin \left(\frac{4 \pi \tau}{\sigma^{2} t}\right) \sin \left(\frac{4 \pi \theta}{\sigma^{2}(1-t)}\right) \frac{\sinh (\tau) \sinh (\theta)}{(\cosh (\tau)+\cosh (\theta))^{4}} d \tau d \theta
\end{aligned}
$$

From the above equation, it follows that the correlation function is singular at $t \rightarrow 0$, and $t \rightarrow 1$. Its form is presented at figure 1 .

At large $T=\sigma t(T \gg 1),(3.36)$ is reduced to

$$
G_{2}(0, T) \approx J(T(\sigma-T))^{-\frac{3}{2}}, \quad J=\frac{4 \sigma}{\pi^{2}} \int_{1}^{+\infty} \int_{1}^{+\infty} \frac{1}{(x+y)^{2}} \frac{d x}{\sqrt{x^{2}-1}} \frac{d y}{\sqrt{y^{2}-1}},
$$

demonstrating the $T^{-\frac{3}{2}}$ behaviour known from [23, 25], although with the different factor.

Note that the functional integrals with any function of $\varphi(t)$ and $\left.\varphi^{\prime}(t), \varphi^{\prime}(0)\right)$ in the integrand of (3.16) can be calculated in exactly the same way. 


\subsection{N-point correlation functions}

The method described in detail in the above subsection can be used to evaluate the N-point correlation function given by the functional integral

$$
\begin{aligned}
& G_{N}^{\alpha}\left(0, t_{1}, \ldots t_{N-1}\right) \\
& =\int_{\operatorname{Diff}^{1}([0,1])} \varphi^{\prime}(0) \varphi^{\prime}\left(t_{1}\right) \ldots \varphi^{\prime}\left(t_{N-1}\right) \delta\left(\frac{\varphi^{\prime}(1)}{\varphi^{\prime}(0)}-1\right) \exp \left\{\frac{2 \alpha^{2}}{\sigma^{2}} \int_{0}^{1}\left(\varphi^{\prime}(\tau)\right)^{2} d \tau\right\} \mu_{\sigma}(d \varphi) .
\end{aligned}
$$

Let

$$
t_{0}=0<t_{1}<\ldots<t_{N-1}<t_{N}=1 ; \quad x_{0}=0, x_{N}=1,
$$

and denote

$\Delta t_{n}=t_{n}-t_{n-1}, \quad \Delta x_{n}=x_{n}-x_{n-1} ; \quad \chi_{\alpha}\left(x_{n}\right)=\frac{\frac{2}{\alpha} \tan \frac{\alpha}{2}}{1+\tan ^{2} \frac{\alpha}{2}\left(2 x_{n}-1\right)^{2}}, \quad n=1, \ldots N$.

Then the N-point correlation function is written as

$$
\begin{aligned}
G_{N}^{\alpha} & \left(0, t_{1}, \ldots t_{N-1}\right)=\left(\Delta t_{1} \cdots \Delta t_{N}\right)^{2} \\
& \times \int_{0}^{1} \chi_{\alpha}\left(x_{N-1}\right) d x_{N-1} \int_{0}^{x_{N-1}} \chi_{\alpha}\left(x_{N-2}\right) d x_{N-2} \cdots \int_{0}^{x_{2}} \chi_{\alpha}\left(x_{1}\right) d x_{1} \frac{1}{\left(\Delta x_{1} \cdots \Delta x_{N}\right)^{3}} \\
& \times \int_{0}^{+\infty} V_{1}^{2} d V_{1} \cdots \int_{0}^{+\infty} V_{N}^{2} d V_{N} \exp \left\{\frac{8 \sin ^{2} \frac{\alpha}{2}}{\sigma^{2}} V_{N}\right\} \mathcal{E}_{\sigma \sqrt{\Delta t_{1}}}\left(\frac{\Delta t_{1}}{\Delta x_{1}} V_{N}, \frac{\Delta t_{1}}{\Delta x_{1}} V_{1}\right) \\
& \times \mathcal{E}_{\sigma \sqrt{\Delta t_{2}}}\left(\frac{\Delta t_{2}}{\Delta x_{2}} V_{1}, \frac{\Delta t_{2}}{\Delta x_{2}} V_{2}\right) \cdots \mathcal{E}_{\sigma \sqrt{\Delta t_{N}}}\left(\frac{\Delta t_{N}}{\Delta x_{N}} V_{N-1}, \frac{\Delta t_{N}}{\Delta x_{N}} V_{N}\right) .
\end{aligned}
$$

Now we can perform functional integration in the functions $\mathcal{E}$, and obtain

$$
\begin{aligned}
& G_{N}^{\alpha}\left(0, t_{1}, \ldots t_{N-1}\right)=\left(\frac{2}{\pi}\right)^{\frac{3}{2} N} \frac{\sigma^{N}}{\sqrt{\Delta t_{1} \cdots \Delta t_{N}}} \exp \left\{\frac{2 \pi^{2}}{\sigma^{2}}\left[\frac{1}{\Delta t_{1}}+\cdots+\frac{1}{\Delta t_{N}}\right]\right\} \\
& \times \int_{0}^{1} \chi_{\alpha}\left(x_{N-1}\right) d x_{N-1} \int_{0}^{x_{N-1}} \chi_{\alpha}\left(x_{N-2}\right) d x_{N-2} \cdots \int_{0}^{x_{2}} \chi_{\alpha}\left(x_{1}\right) d x_{1} \frac{1}{\left(\Delta x_{1} \cdots \Delta x_{N}\right)^{2}} \\
& \times \int_{0}^{+\infty} d U_{1} \cdots \int_{0}^{+\infty} d U_{N} \int_{0}^{+\infty} d \tau_{1} \cdots \int_{0}^{+\infty} d \tau_{N} U_{1} \cdots U_{N} \exp \left\{8 \sin ^{2} \frac{\alpha}{2} U_{N}\right\} \\
& \times \exp \left\{-\frac{2}{\Delta x_{1}}\left(U_{N}+U_{1}\right)-\frac{2}{\Delta x_{2}}\left(U_{1}+U_{2}\right)-\ldots-\frac{2}{\Delta x_{N}}\left(U_{N-1}+U_{N}\right)\right\} \\
& \times \exp \left\{-\frac{4}{\Delta x_{1}} \sqrt{U_{N} U_{1}} \cosh \tau_{1}-\frac{4}{\Delta x_{2}} \sqrt{U_{1} U_{2}} \cosh \tau_{2}-\ldots-\frac{4}{\Delta x_{N}} \sqrt{U_{N-1} U_{N}} \cosh \tau_{N}\right\} \\
& \times \exp \left\{-\frac{2 \tau_{1}^{2}}{\sigma^{2} \Delta t_{1}}\right\} \sin \left(\frac{4 \pi \tau_{1}}{\sigma^{2} \Delta t_{1}}\right) \sinh \tau_{1} \cdots \exp \left\{-\frac{2 \tau_{N}^{2}}{\sigma^{2} \Delta t_{N}}\right\} \sin \left(\frac{4 \pi \tau_{N}}{\sigma^{2} \Delta t_{N}}\right) \sinh \tau_{N} .
\end{aligned}
$$


The analysis of the dependence of the function $G_{N}^{\alpha}$ on the regularization parameter $\alpha$ as well as the study of possible relations between different correlation functions will be given in another paper. Here, we only note that $G_{N}\left(0, t_{1}, \ldots t_{N-1}\right)$ is singular if there is a pair of coinciding arguments, that is, when some $\Delta t_{n}=0$, similarly to the equation (3.36).

\section{Concluding remarks}

Now the Schwarzian theory has a growing number of physical applications. (Besides the references cited in the introduction, see also the recent papers [38, 39].) Therefore, it is important for the physicists working in this field to have a regular method of doing calculations.

In this paper, we propose a universal method of the explicit evaluating functional integrals over the quasi-invariant measures on the infinite-dimensional groups, and evaluate the functional integrals assigning correlation functions in the Schwarzian theory. The great merit of the method is that it reduces the problem of the evaluation of various functional integrals to the evaluation of the functional integral (3.26) only. All other functional integrals in this theory are represented as ordinary integrals with the functions $\mathcal{E}_{\sigma}(u, v)$ in the integrands.

In contrast to the papers [23-26], we integrate over the arbitrary diffeomorphisms and keep the $\operatorname{SL}(2, \mathbf{R})$ invariance (at $\alpha \rightarrow \pi$ ) at any step of the calculations. Then we divide (normalize) the result of the functional integration by the corresponding integral over the $\operatorname{SL}(2, \mathbf{R})$ group. Thus we factor the infinite input of the $\operatorname{SL}(2, \mathbf{R})$ subgroup out.

In some sense, the method of [23-26] looks like calculations in a gauge theory using the transverse gauge, whereas our approach is more similar to calculations in an arbitrary gauge with a gauge fixing term in the action.

In spite of their difference, the both approaches lead to qualitatively the same results for the two point correlation function. Namely, the form of the function given above at figure 1 is similar to that given in [25] at figure 6 . The both results also have the same $T^{-\frac{3}{2}}$ behaviour at $T \gg 1$. Thus we confirm the corresponding physical conclusions of the earlier papers [23-26].

\section{A A quasi-invariant measure on the group of diffeomorphisms}

In this appendix, we define the measure on the group of diffeomorphisms and give a schematic proof of its quasi-invariance.

Let $\operatorname{Diff}_{+}^{1}([0,1])$ be the group of all continuously differentiable transformations of the interval $[0,1]$ preserving the ends, and $\operatorname{Diff}_{+}^{3}([0,1])$ be the subgroup of the group Diff ${ }_{+}^{1}([0,1])$ consisting of all diffeomorphisms of the smoothness $C^{3}$.

Denote the space of all continuous functions on the interval $[0,1]$ with zero value at the left end of the interval by $C_{0}([0,1])$.

Consider the map

$$
A: \operatorname{Diff}_{+}^{1}([0,1]) \rightarrow C_{0}([0,1])
$$


where

$$
\xi(t)=(A(\varphi))(t)=\log \left(\varphi^{\prime}(t)\right)-\log \left(\varphi^{\prime}(0)\right), \quad \forall t \in[0,1] .
$$

The map $A$ identifies the spaces $\operatorname{Diff}_{+}^{1}([0,1])$ and $C_{0}([0,1])$. In this case,

$$
A^{-1}(\xi)(t)=\frac{\int_{0}^{t} e^{\xi(\tau)} d \tau}{\int_{0}^{1} e^{\xi(\tau)} d \tau} .
$$

Let $w_{\sigma}$ be the Wiener measure with the dispersion $\sigma$ on $C_{0}([0,1])$.

Now define the measure $\mu_{\sigma}$ on $\operatorname{Diff}_{+}^{1}([0,1])$ by the equation $\mu_{\sigma}(X)=w_{\sigma}(A(X))$ for any measurable subset $X$ of the space $\operatorname{Diff}_{+}^{1}([0,1])$.

For every $f \in \operatorname{Diff}_{+}^{3}([0,1])$ and an arbitrary $\varphi \in \operatorname{Diff}_{+}^{1}([0,1])$ define

$$
L_{f}(\varphi)=f \circ \varphi .
$$

A detailed proof of the quasi-invariance of the measure $\mu_{\sigma}$ is given in [36]). Here, we present a scheme of the proof.

Consider

$$
\eta=A L_{f} A^{-1}(\xi)
$$

It is written in the form

$$
\eta(t)=\xi(t)+h\left(\frac{\int_{0}^{t} e^{\xi(\tau)} d \tau}{\int_{0}^{1} e^{\xi(\tau)} d \tau}\right),
$$

where $h=A(f)$, that is,

$$
h(t)=\log \left(f^{\prime}(t)\right)-\log \left(f^{\prime}(0)\right) .
$$

Note that, if $\varphi \in \operatorname{Diff}_{+}^{3}([0,1])$, then $h \in C_{0}^{2}([0,1])$.

The Jacobian of the map (A.3) at the point $\xi$ found in the [36]) does not depend on $\xi$ and is equal to $\frac{1}{f^{\prime}(1)}$.

For continuously differentiable function $\eta$, we obtain

$$
\int_{0}^{1}\left(\eta^{\prime}(t)\right)^{2} d t=\int_{0}^{1}\left(\xi^{\prime}(t)\right)^{2} d t+W+V
$$

where

$$
\begin{aligned}
& W=2 \int_{0}^{1} \xi^{\prime}(t) h^{\prime}\left(\frac{\int_{0}^{t} e^{\xi(\tau)} d \tau}{\int_{0}^{1} e^{\xi(\tau)} d \tau}\right) \frac{e^{\xi(t)}}{\int_{0}^{1} e^{\xi(\tau)} d \tau} d t, \\
& V=\int_{0}^{1}\left(h^{\prime}\left(\frac{\int_{0}^{t} e^{\xi(\tau)} d \tau}{\int_{0}^{1} e^{\xi(\tau)} d \tau}\right)\right)^{2} \frac{e^{2 \xi(t)}}{\left(\int_{0}^{1} e^{\xi(\tau)} d \tau\right)^{2}} d t .
\end{aligned}
$$


Let $P$ be a continuous bounded functional on $C_{0}([0,1])$. Then

$$
\int_{C_{0}([0,1])} P(\eta) w_{\sigma}(d \eta)=\frac{1}{f^{\prime}(1)} \int_{C_{0}([0,1])} P\left(A L_{f} A^{-1}(\xi)\right) \exp \left\{-\frac{1}{2 \sigma^{2}}(W+V)\right\} w_{\sigma}(d \xi) .
$$

Note that if $\xi$ were continuously differentiable, then by the integration by parts we would get

$$
\frac{1}{2} W=h^{\prime}(1) \frac{e^{\xi(1)}}{\int_{0}^{1} e^{\xi(\tau)} d \tau}-h^{\prime}(0) \frac{1}{\int_{0}^{1} e^{\xi(\tau)} d \tau}-\int_{0}^{1} h^{\prime \prime}\left(\frac{\int_{0}^{t} e^{\xi(\tau)} d \tau}{\int_{0}^{1} e^{\xi(\tau)} d \tau}\right) \frac{e^{2 \xi(t)}}{\left(\int_{0}^{1} e^{\xi(\tau)} d \tau\right)^{2}} d t .
$$

However, the Wiener process $\xi(t)$ is nonsmooth, and there appear the additional terms [40] that can be evaluated in the discrete version of the theory by the correct passage to the continuous limit (see [36]).

As the result, we have

$$
\begin{aligned}
& \int_{C_{0}([0,1])} P(\eta) w_{\sigma}(d \eta) \\
& =\frac{1}{\sqrt{f^{\prime}(1) f^{\prime}(1)}} \int_{C_{0}([0,1])} P\left(A L_{f} A^{-1} \xi\right) \exp \left\{-\frac{1}{2 \sigma^{2}}\left(h^{\prime}(1) \frac{e^{\xi(1)}}{\int_{0}^{1} e^{\xi(\tau)} d \tau}-h^{\prime}(0) \frac{1}{\int_{0}^{1} e^{\xi(\tau)} d \tau}\right)\right\} \\
& \quad \times \exp \left\{\frac{1}{\sigma^{2}} \int_{0}^{1} \mathcal{S}_{f}\left(\frac{\int_{0}^{t} e^{\xi(\tau)} d \tau}{\int_{0}^{1} e^{\xi(\tau)} d \tau}\right) \frac{e^{2 \xi(t)}}{\left(\int_{0}^{1} e^{\xi(\tau)} d \tau\right)^{2}} d t\right\} w_{\sigma}(d \xi) .
\end{aligned}
$$

Note that

$$
\mathcal{S}_{f}(t)=\left(\frac{f^{\prime \prime}(t)}{f^{\prime}(t)}\right)^{\prime}-\frac{1}{2}\left(\frac{f^{\prime \prime}(t)}{f^{\prime}(t)}\right)^{2}=h^{\prime \prime}(t)-\frac{1}{2}(h(t))^{2} .
$$

The map $A^{-1}$ gives the equation (2.8)

$$
\begin{aligned}
& \int_{\operatorname{Diff}^{1}([0,1])} F(\varphi) \mu_{\sigma}(d \varphi)=\frac{1}{\sqrt{f^{\prime}(0) f^{\prime}(1)}} \int_{\operatorname{Diff}^{1}([0,1])} F(f \circ \varphi) \\
& \quad \times \exp \left\{\frac{1}{\sigma^{2}}\left[\frac{f^{\prime \prime}(0)}{f^{\prime}(0)} \varphi^{\prime}(0)-\frac{f^{\prime}(1)}{f^{\prime}(1)} \varphi^{\prime}(1)\right]+\frac{1}{\sigma^{2}} \int_{0}^{1} \mathcal{S}_{f}(\varphi(t))\left(\varphi^{\prime}(t)\right)^{2} d t\right\} \mu_{\sigma}(d \varphi) .
\end{aligned}
$$

Thus, the measure $\mu_{\sigma}$ on $\operatorname{Diff}_{+}^{1}([0,1])$ is quasi-invariant with respect to the subgroup $\operatorname{Diff}_{+}^{3}([0,1])$ :

$$
\mu_{\sigma}\left(L_{f}(X)\right)=\int_{X} \mathcal{R}_{f}^{\mu}(\varphi) \mu_{\sigma}(d \varphi)
$$


where the Radon - Nikodim derivative has the form (2.7)

$$
\begin{aligned}
\mathcal{R}_{f}^{\mu}(\varphi) \equiv \frac{d \mu_{\sigma}^{f}}{d \mu_{\sigma}}(\varphi)= & \frac{1}{\sqrt{f^{\prime}(0) f^{\prime}(1)}} \\
& \times \exp \left\{\frac{1}{\sigma^{2}}\left[\frac{f^{\prime \prime}(0)}{f^{\prime}(0)} \varphi^{\prime}(0)-\frac{f^{\prime \prime}(1)}{f^{\prime}(1)} \varphi^{\prime}(1)\right]+\frac{1}{\sigma^{2}} \int_{0}^{1} \mathcal{S}_{f}(\varphi(t))\left(\varphi^{\prime}(t)\right)^{2} d t\right\} .
\end{aligned}
$$

\section{B Proof of the basic formula}

To evaluate the basic Wiener integral (2.2) we use the quasi-invariance of the Wiener measure under the action of the operator $K_{f} \equiv A L_{f} A^{-1}$ :

$$
\left(K_{f} \xi\right)(t)=\xi(t)+\log \left\{f^{\prime}\left(\frac{\int_{0}^{t} e^{\xi(\tau)} d \tau}{\int_{0}^{1} e^{\xi(\tau)} d \tau}\right)\right\}-\log \left\{f^{\prime}(0)\right\} .
$$

The Radon-Nikodim derivative is

$$
\begin{aligned}
\frac{d w_{\sigma}^{f}}{d w_{\sigma}}(\xi)= & \frac{1}{\sqrt{f^{\prime}(0) f^{\prime}(1)}} \exp \left\{\frac{1}{\sigma^{2}}\left[\frac{f^{\prime \prime}(0)}{f^{\prime}(0)}-\frac{f^{\prime \prime}(1)}{f^{\prime}(1)} e^{\xi(1)}\right] \frac{1}{\int_{0}^{1} e^{\xi(\tau)} d \tau}\right\} \\
& \times \exp \left\{\frac{1}{\sigma^{2}} \int_{0}^{1} \mathcal{S}_{f}\left(\frac{\int_{0}^{t} e^{\xi(\tau)} d \tau}{\int_{0}^{1} e^{\xi(\tau)} d \tau}\right) \frac{e^{2 \xi(t)}}{\left(\int_{0}^{1} e^{\xi(\tau)} d \tau\right)^{2}} d t\right\}
\end{aligned}
$$

where $w_{\sigma}^{f}(X)=w_{\sigma}\left(K_{f} X\right)$.

Now consider the special transformation

$$
f=g_{\beta}(t)=\frac{(\beta+1) t}{\beta t+1}
$$

with

$$
g_{\beta}^{\prime}(t)=\frac{\beta+1}{(\beta t+1)^{2}}, \quad g_{\beta}^{\prime \prime}(t)=-\frac{2(\beta+1) \beta}{(\beta t+1)^{3}}, \quad \mathcal{S}_{g_{\beta}}(t)=0
$$

In this case, the Radon-Nikodim derivative has the form

$$
\frac{d w_{\sigma}^{g_{\beta}}}{d w_{\sigma}}(\xi)=\exp \left\{\frac{-2 \beta}{\sigma^{2}}\left[1-\frac{e^{\xi(1)}}{\beta+1}\right] \frac{1}{\int_{0}^{1} e^{\xi(\tau)} d \tau}\right\}
$$


and

$\int_{C_{0}([0,1])} \delta(\xi(1)-x) w_{\sigma}^{g_{\beta}}(d \xi)=\int_{C_{0}([0,1])} \delta(\xi(1)-x) \exp \left\{\frac{-2 \beta}{\sigma^{2}}\left[1-\frac{e^{\xi(1)}}{\beta+1}\right] \frac{1}{\int_{0}^{1} e^{\xi(\tau)} d \tau}\right\} w_{\sigma}(d \xi)$.

At the same time,

$$
\begin{aligned}
\int_{C_{0}([0,1])} \delta(\xi(1)-x) w_{\sigma}^{g_{\beta}}(d \xi) & =\int_{C_{0}([0,1])} \delta(\eta(1)+2 \log (\beta+1)-x) w_{\sigma}(d \eta) \\
& =\frac{1}{\sqrt{2 \pi} \sigma} \exp \left\{-\frac{1}{2 \sigma^{2}}(x-2 \log (\beta+1))^{2}\right\} .
\end{aligned}
$$

Here, we have used the equation

$$
\int_{C_{0}([0,1])} \delta(\xi(1)-x) w_{\sigma}(d \xi)=\frac{1}{\sqrt{2 \pi} \sigma} \exp \left\{-\frac{1}{2 \sigma^{2}} x^{2}\right\},
$$

and the following relation:

$$
\eta(1)=\left(K_{g_{\beta}} \xi\right)(1)=\xi(1)+\log g_{\beta}^{\prime}(1)-\log g_{\beta}^{\prime}(0)=\xi(1)-2 \log (\beta+1),
$$

or

$$
\xi(1)=\eta(1)+2 \log (\beta+1) .
$$

Therefore, the basic formula

$$
\begin{aligned}
\int_{C_{0}([0,1])} \delta(\xi(1)-x) \exp & \left\{\frac{-2 \beta}{\sigma^{2}}\left[1-\frac{e^{\xi(1)}}{\beta+1}\right] \frac{1}{\int_{0}^{1} e^{\xi(\tau)} d \tau}\right\} w_{\sigma}(d \xi) \\
& =\frac{1}{\sqrt{2 \pi} \sigma} \exp \left\{-\frac{1}{2 \sigma^{2}}(x-2 \log (\beta+1))^{2}\right\}
\end{aligned}
$$

is proven.

\section{Properties of the function $\mathcal{E}_{\sigma}(u, v)$}

To study the properties of the function $\mathcal{E}_{\sigma}(u, v)$ given by the equation (3.26)

$$
\mathcal{E}_{\sigma}(u, v)=\int_{\operatorname{Diff}^{1}([0,1])} \delta\left(\varphi^{\prime}(0)-u\right) \delta\left(\varphi^{\prime}(1)-v\right) \mu_{\sigma}(d \varphi)
$$

consider the functions $\varphi, \psi \in \operatorname{Diff}^{1}([0,1])$ connected by the diffeomorphism $g_{\lambda}$ :

$$
\varphi(t)=g_{\lambda}(\psi(t)), \quad g_{\lambda}(\tau)=\frac{(\lambda-1) \tau}{\lambda-\tau}, \quad \lambda>1, \quad g_{\lambda} \in \operatorname{Diff}^{3}([0,1]) .
$$


In this case, the equation (2.8) gives

$$
\begin{aligned}
\mathcal{E}_{\sigma}(u, v)= & \int_{\operatorname{Diff}([0,1])} \delta\left(g_{\lambda}^{\prime}(0) \psi^{\prime}(0)-u\right) \delta\left(g_{\lambda}^{\prime}(1) \psi^{\prime}(1)-v\right) \\
& \times \exp \left\{\frac{1}{\sigma^{2}}\left[\frac{g^{\prime \prime}(0)}{g^{\prime}(0)} \psi^{\prime}(0)-\frac{g^{\prime \prime}(1)}{g^{\prime}(1)} \psi^{\prime}(1)\right]\right\} \mu_{\sigma}(d \psi) \\
= & \int_{\operatorname{Diff}^{1}([0,1])} \delta\left(\psi^{\prime}(0)-\frac{\lambda}{\lambda-1} u\right) \delta\left(\psi^{\prime}(1)-\frac{\lambda-1}{\lambda} v\right) \exp \left\{\frac{2}{\sigma^{2}}\left(\frac{u}{\lambda-1}-\frac{v}{\lambda}\right)\right\} \mu_{\sigma}(d \psi) .
\end{aligned}
$$

Therefore, we have

$$
\mathcal{E}_{\sigma}(u, v)=\mathcal{E}_{\sigma}\left(\frac{\lambda}{\lambda-1} u, \frac{\lambda-1}{\lambda} v\right) \exp \left\{\frac{2}{\sigma^{2}}\left(\frac{u}{\lambda-1}-\frac{v}{\lambda}\right)\right\} .
$$

In particular, for

$$
\frac{1}{\lambda}=1-\sqrt{\frac{u}{v}},
$$

the above equation has the symmetric form (3.29):

$$
\mathcal{E}_{\sigma}(u, v)=\mathcal{E}_{\sigma}(\sqrt{u v}, \sqrt{u v}) \exp \left\{-\frac{2}{\sigma^{2}}(\sqrt{u}-\sqrt{v})^{2}\right\} .
$$

Now we perform the functional integration in $\mathcal{E}_{\sigma}(u, u)$. First we write it in the form of the integrals over the Wiener measure

$$
\begin{aligned}
\mathcal{E}_{\sigma}(u, u) & =\int_{\operatorname{Diff}^{1}([0,1])} \delta\left(\varphi^{\prime}(0)-u\right) \delta\left(\varphi^{\prime}(1)-u\right) \mu_{\sigma}(d \varphi) \\
& =\int_{\operatorname{Diff}^{1}([0,1])} \delta\left(\varphi^{\prime}(0)-u\right) \delta\left(\varphi^{\prime}(1)-\varphi^{\prime}(0)\right) \mu_{\sigma}(d \varphi) \\
& =\int_{C_{0}([0,1])} \delta\left(\frac{1}{\int_{0}^{1} e^{\xi(\tau)} d \tau}-u\right) \delta\left(u\left[e^{\xi(1)}-1\right]\right) w_{\sigma}(d \xi) \\
& =\frac{1}{u} \int_{C_{0}([0,1])} \delta\left(\frac{1}{\int_{0}^{1} e^{\xi(\tau)} d \tau}-u\right) \delta(\xi(1)) w_{\sigma}(d \xi) .
\end{aligned}
$$

Taking the Fourier transform of the first $\delta$-function in (C.4), we get

$$
\mathcal{E}_{\sigma}(u, u)=\frac{1}{2 \pi u} \int_{-\infty}^{+\infty} e^{i \rho u} d \rho \int_{C_{0}([0,1])} \exp \left\{-i \rho \frac{1}{\int_{0}^{1} e^{\xi(\tau)} d \tau}\right\} \delta(\xi(1)) w_{\sigma}(d \xi)
$$


It is convenient to rewrite the above equation as

$$
\mathcal{E}_{\sigma}(u, u)=\frac{1}{u \pi \sigma^{2}} \int_{-\infty}^{+\infty} \exp \left\{i u \frac{2 r}{\sigma^{2}}\right\} d r \int_{C_{0}([0,1])} \exp \left\{-i \frac{2 r}{\sigma^{2}} \frac{1}{\int_{0}^{1} e^{\xi(\tau)} d \tau}\right\} \delta(\xi(1)) w_{\sigma}(d \xi) .
$$

To use the equation (2.1), note that a solution of the equation

$$
\frac{\beta^{2}}{\beta+1}=i r
$$

has the form

$$
\beta_{*}(r)+1=\left(i \frac{r}{2}+1\right)+\sqrt{\left(i \frac{r}{2}+1\right)^{2}-1} .
$$

Due to the identity

$$
\log \left(y+\sqrt{y^{2}-1}\right)=\operatorname{arccosh} y,
$$

the equation $(2.1)$ gives

$$
\int_{C_{0}([0,1])} \exp \left\{-i \frac{2 r}{\sigma^{2}} \frac{1}{\int_{0}^{1} e^{\xi(\tau)} d \tau}\right\} \delta(\xi(1)) w_{\sigma}(d \xi)=\frac{1}{\sqrt{2 \pi} \sigma} \exp \left\{-\frac{2}{\sigma^{2}}\left(\operatorname{arccosh}\left(\frac{i r}{2}+1\right)\right)^{2}\right\} .
$$

Therefore, the function $\mathcal{E}_{\sigma}(u, u)$ is written as

$$
\mathcal{E}_{\sigma}(u, u)=\frac{2}{u(2 \pi)^{\frac{3}{2}} \sigma^{3}} \int_{-\infty}^{+\infty} \exp \left\{i u \frac{2 r}{\sigma^{2}}\right\} \exp \left\{-\frac{2}{\sigma^{2}}\left(\operatorname{arccosh}\left(\frac{i r}{2}+1\right)\right)^{2}\right\} d r .
$$

Having in mind the properties of the function arccosh, we turn the integration contour with the result

$$
\begin{aligned}
\mathcal{E}_{\sigma}(u, u)= & \frac{\sqrt{2}}{u(2 \pi)^{\frac{3}{2}} \sigma^{3}} \exp \left\{\frac{2 \pi^{2}}{\sigma^{2}}\right\} \int_{4}^{+\infty} \exp \left\{-\frac{2 u x}{\sigma^{2}}\right\} \\
& \times \exp \left\{-\frac{2}{\sigma^{2}}\left(\operatorname{arccosh}\left(\frac{x-2}{2}\right)\right)^{2}\right\} \sin \left(\frac{4 \pi}{\sigma^{2}} \operatorname{arccosh}\left(\frac{x-2}{2}\right)\right) d x .
\end{aligned}
$$

After the substitution

$$
\tau=\operatorname{arccosh}\left(\frac{x-2}{2}\right), \quad x=2+2 \cosh \tau,
$$

the function $\mathcal{E}_{\sigma}(u, u)$ takes the form (3.30)

$$
\mathcal{E}_{\sigma}(u, u)=\frac{2 \sqrt{2}}{u \pi^{\frac{3}{2}} \sigma^{3}} \exp \left\{\frac{2 \pi^{2}-4 u}{\sigma^{2}}\right\} \int_{0}^{+\infty} \exp \left\{-\frac{2}{\sigma^{2}}\left(2 u \cosh \tau+\tau^{2}\right)\right\} \sin \left(\frac{4 \pi \tau}{\sigma^{2}}\right) \sinh (\tau) d \tau .
$$

The forms of the functions $\mathcal{E}_{\sigma}(x, x)$ and $\mathcal{E}_{\sigma}(x, y)$ at $\sigma=1$ are presented at figure 2 and at figure 3 respectively. 


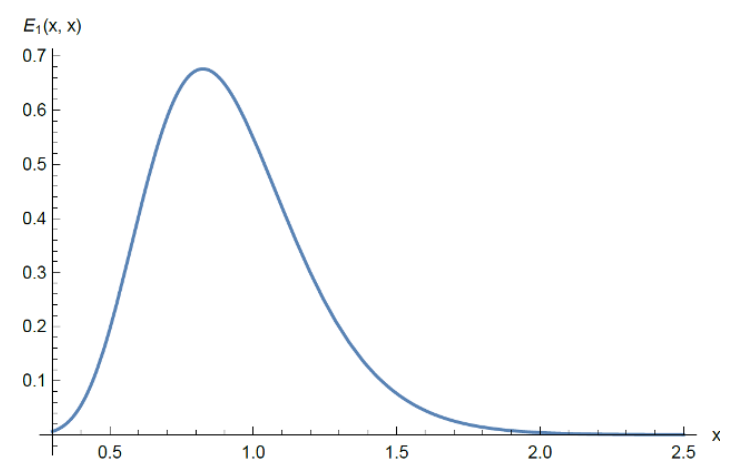

Figure 2. The form of the symmetric function $\mathcal{E}_{1}(x, x)$.

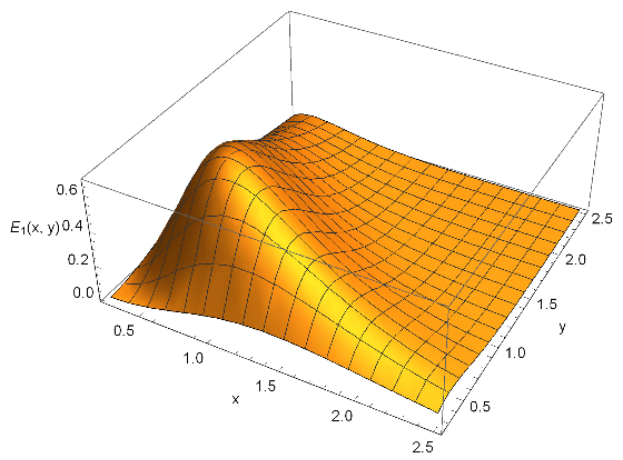

Figure 3. The form of the function $\mathcal{E}_{\sigma}(x, y)$.

\section{Integration in $\mathrm{SL}(2, \mathrm{R})$}

In this appendix, we evaluate the asymptotic form (at $\pi-\alpha \rightarrow 0$ ) of the regularized integrals over the Haar measure $d \nu$ on the group $\mathrm{SL}(2, \mathbf{R})$. These integrals are used to normalize the corresponding functional integrals over the group Diff ${ }^{1}$ considered in this paper. We are interesting in the asymptotic form of the following integrals:

$$
\begin{aligned}
V_{\mathrm{SL}(2, \mathbf{R})}^{\alpha} & =\int_{\mathrm{SL}(2, \mathbf{R})} \exp \left\{-\lambda \int_{0}^{1} \dot{\varphi}^{2}(t) d t\right\} d \nu, \\
\Phi_{\mathrm{SL}(2, \mathbf{R})}^{\alpha} & =\int_{\mathrm{SL}(2, \mathbf{R})} \dot{\varphi}(0) \exp \left\{-\lambda \int_{0}^{1} \dot{\varphi}^{2}(t) d t\right\} d \nu, \\
G_{2 ; \mathrm{SL}(2, \mathbf{R})}^{\alpha} & =\int_{\mathrm{SL}(2, \mathbf{R})} \dot{\varphi}(t) \dot{\varphi}(0) \exp \left\{-\lambda \int_{0}^{1} \dot{\varphi}^{2}(t) d t\right\} d \nu .
\end{aligned}
$$

Here,

$$
\lambda=\frac{2\left[\pi^{2}-\alpha^{2}\right]}{\sigma^{2}} .
$$


To perform the integration over the group $\mathrm{SL}(2, \mathbf{R})$ we choose the representation [41]

$$
\varphi_{z}(t)=-\frac{i}{2 \pi} \log \frac{e^{i 2 \pi t}+z}{\bar{z} e^{i 2 \pi t}+1}, \quad z=\rho e^{i \theta}, \quad \rho<1 .
$$

In this case, the Haar measure is [41]

$$
\nu(d z)=\frac{2 d z d \bar{z}}{(1-z \bar{z})^{2}}=\frac{4 \rho d \rho d \theta}{\left(1-\rho^{2}\right)^{2}} .
$$

To evaluate the integral

$$
I=\int_{0}^{1} \dot{\varphi}_{z}^{2}(t) d t=\left(1-|z|^{2}\right)^{2} \int_{0}^{1} \frac{d t}{\left(e^{i 2 \pi t}+z\right)^{2}\left(e^{-i 2 \pi t}+\bar{z}\right)^{2}}
$$

note that, due to the periodicity, it does not depend on $\theta$. Therefore, we can assume $z$ to be real $z=\rho>0, \rho<1$. After the substitution $w=\rho \exp \{i 2 \pi t\}$, the integral $I$ transforms into the contour integral

$$
\begin{aligned}
I & =\frac{1}{2 \pi i}\left(1-\rho^{2}\right)^{2} \oint_{|w|=\rho<1} \frac{w d w}{\left(w+\rho^{2}\right)^{2}(w+1)^{2}} \\
& =\left(1-\rho^{2}\right)^{2} \operatorname{Res}_{\left\{w=-\rho^{2}\right\}} \frac{w}{\left(w+\rho^{2}\right)^{2}(w+1)^{2}}=-1+\frac{2}{1-\rho^{2}}=-1+\frac{2}{1-z \bar{z}} .
\end{aligned}
$$

And the regularized volume of the group (D.1) has the form

$$
V_{\mathrm{SL}(2, \mathbf{R})}^{\alpha}=e^{\lambda} \int_{|z|<1} \exp \left\{-\lambda \frac{2}{(1-z \bar{z})}\right\} \frac{2 d z d \bar{z}}{(1-z \bar{z})^{2}}=e^{\lambda} \int_{0}^{1} \exp \left\{-\lambda \frac{2}{\left(1-\rho^{2}\right)}\right\} \frac{8 \pi \rho d \rho}{\left(1-\rho^{2}\right)^{2}}=\frac{2 \pi}{\lambda}
$$

Thus, at $\alpha \rightarrow \pi-0$,

$$
V_{\mathrm{SL}(2, \mathbf{R})}^{A s}=\frac{\sigma^{2}}{2(\pi-\alpha)} .
$$

To find the second integral

$$
\Phi_{\mathrm{SL}(2, \mathbf{R})}^{\alpha}=e^{\lambda} \int_{|z|<1} \exp \left\{-\lambda \frac{2}{(1-z \bar{z})}\right\} \frac{1}{(1+z)(1+\bar{z})} \frac{2 d z d \bar{z}}{(1-z \bar{z})}
$$

it is convenient to use the following representation of the complex variable $z$ :

$$
z=-1+\varrho e^{i \vartheta}
$$

and write (D.2) in the form

$$
\Phi_{\mathrm{SL}(2, \mathbf{R})}^{\alpha}=2 e^{\lambda} \int_{-\frac{\pi}{2}}^{+\frac{\pi}{2}} d \vartheta \int_{0}^{2 \cos \vartheta} \exp \left\{-\frac{2 \lambda}{\varrho(2 \cos \vartheta-\varrho)}\right\} \frac{d \varrho}{\varrho^{2}(2 \cos \vartheta-\varrho)} .
$$


Note that in terms of $\varrho$ and $\vartheta$, the integral in (D.1) looks like

$$
V_{\mathrm{SL}(2, \mathbf{R})}^{\alpha}=2 e^{\lambda} \int_{-\frac{\pi}{2}}^{+\frac{\pi}{2}} d \vartheta \int_{0}^{2 \cos \vartheta} \exp \left\{-\frac{2 \lambda}{\varrho(2 \cos \vartheta-\varrho)}\right\} \frac{d \varrho}{\varrho(2 \cos \vartheta-\varrho)^{2}} .
$$

The substitution

$$
\tilde{\varrho}=2 \cos \vartheta-\varrho
$$

transforms the integral (D.10) into the integral (D.11).

Thus we have

$$
\Phi_{\mathrm{SL}(2, \mathbf{R})}^{A s}=\frac{\sigma^{2}}{2(\pi-\alpha)} .
$$

Now we find the asymptotic form of the integral

$$
\begin{aligned}
G_{2 ; \operatorname{SL}(2, \mathbf{R})}^{\alpha}\left(0, \frac{1}{2}\right) & =e^{\lambda} \int_{|z|<1} \exp \left\{-\lambda \frac{2}{(1-z \bar{z})}\right\} \frac{2 d z d \bar{z}}{(1+z)(1+\bar{z})(1-z)(1-\bar{z})} \\
& =e^{\lambda} \int_{0}^{2 \pi} d \theta \int_{0}^{1} \exp \left\{-\lambda \frac{2}{\left(1-\rho^{2}\right)}\right\} \frac{2 d \rho^{2}}{\left(1-\rho^{2}\right)^{2}+4 \rho^{2} \sin ^{2} \theta} .
\end{aligned}
$$

The method widely used to study the asymptotic behavior of Feynman diagrams (see, e.g., [42] and refs. therein) is very helpful here. Namely, we consider the Mellin transform of the integrand and rewrite (D.13) as follows:

$$
\begin{aligned}
G_{2 ; \operatorname{SL}(2, \mathbf{R})}^{\alpha}\left(0, \frac{1}{2}\right)= & e^{\lambda} \int_{a_{0}-i \infty}^{a_{0}+i \infty} d a \Gamma(a) \Gamma(1-a) \int_{0}^{2 \pi} d \theta\left(\sin ^{2} \theta\right)^{-a} \\
& \times \int_{0}^{1} \exp \left\{-\lambda \frac{2}{\left(1-\rho^{2}\right)}\right\} \frac{2 d \rho^{2}}{4^{a}\left(1-\rho^{2}\right)^{2(1-a)} \rho^{2 a}}, \quad 0<a_{0}<1 .
\end{aligned}
$$

After the substitution $x=\left(1-\rho^{2}\right)^{-1}$, the integrals over $\theta$ and over $x$ are the table integrals (see, e.g., [37] n. 2.5.3.2 and n. 2.3.6.7):

$$
\begin{aligned}
\int_{0}^{\frac{\pi}{2}} \sin ^{-2 a} \theta d \theta & =\frac{1}{2} \frac{\Gamma\left(\frac{1}{2}-a\right) \Gamma\left(\frac{1}{2}\right)}{\Gamma(1-a)}, \\
\int_{1}^{+\infty} x^{-a}(x-1)^{-a} e^{-2 \lambda x} d x & =\frac{e^{-\lambda} \sqrt{\pi} \Gamma(1-a)}{2 \sin \left(\left[\frac{1}{2}-a\right] \pi\right)}(2 \lambda)^{-\frac{1}{2}+a}\left[I_{-\frac{1}{2}+a}(\lambda)-I_{\frac{1}{2}-a}(\lambda)\right] .
\end{aligned}
$$

Here, $I_{\nu}(\lambda)$ is the modified Bessel function

$$
I_{\nu}(\lambda)=\sum_{k=0}^{\infty} \frac{\lambda^{k+\nu}}{k ! 2^{k+\nu} \Gamma(k+\nu+1)} .
$$


Note that the first term in the series $(k=0)$ is the leading one at $\lambda \rightarrow 0$. As the result, the integral is reduced to

$$
G_{2 ; \mathrm{SL}(2, \mathbf{R})}^{\alpha}\left(0, \frac{1}{2}\right)=\pi \int_{a_{0}-i \infty}^{a_{0}+i \infty} d a \frac{\Gamma(a) \Gamma(1-a)}{4^{a} \Gamma\left(\frac{1}{2}+a\right)} \frac{\Gamma\left(\frac{1}{2}-a\right)}{\sin \left(\left[\frac{1}{2}-a\right] \pi\right)} \lambda^{2 a-1} .
$$

Then we close the integration contour in the right-hand half-plane and note that the integral over the infinite half-circle equals to zero.

Now the leading asymptotics of the function $G_{2 ; \operatorname{SL}(2, \mathbf{R})}^{\alpha}\left(0, \frac{1}{2}\right)$ is given by the residue at the pole of the integrand inside the contour with the minimal value of $a$. For (D.15), it is the pole at $a=\frac{1}{2}$ and

$$
G_{2 ; \operatorname{SL}(2, \mathbf{R})}^{A s}\left(0, \frac{1}{2}\right)=-\pi \log (\pi-\alpha)
$$

Open Access. This article is distributed under the terms of the Creative Commons Attribution License (CC-BY 4.0), which permits any use, distribution and reproduction in any medium, provided the original author(s) and source are credited.

\section{References}

[1] A. Kitaev, Hidden correlations in the Hawking radiaion and thermal noise, talk at KITP, February 2015 [http://online.kitp.ucsb.edu/online/joint98/kitaev/].

[2] A. Kitaev, A simple model of quantum holography, talks at KITP, April and May 2015 [http://online.kitp.ucsb.edu/online/entangled15/kitaev/] [http://online.kitp.ucsb.edu/online/entangled15/kitaev2/].

[3] J. Maldacena and D. Stanford, Remarks on the Sachdev-Ye-Kitaev model, Phys. Rev. D 94 (2016) 106002 [arXiv: 1604.07818] [INSPIRE].

[4] J. Polchinski and V. Rosenhaus, The Spectrum in the Sachdev-Ye-Kitaev Model, JHEP 04 (2016) 001 [arXiv: 1601.06768] [inSPIRE].

[5] A. Jevicki, K. Suzuki and J. Yoon, Bi-Local Holography in the SYK Model, JHEP 07 (2016) 007 [arXiv: 1603.06246] [INSPIRE].

[6] A. Jevicki and K. Suzuki, Bi-Local Holography in the SYK Model: Perturbations, JHEP 11 (2016) 046 [arXiv: 1608.07567] [inSPIRE].

[7] D.J. Gross and V. Rosenhaus, A Generalization of Sachdev-Ye-Kitaev, JHEP 02 (2017) 093 [arXiv: 1610.01569] [INSPIRE].

[8] D.J. Gross and V. Rosenhaus, The Bulk Dual of SYK: Cubic Couplings, JHEP 05 (2017) 092 [arXiv: 1702. 08016] [INSPIRE].

[9] S.R. Das, A. Jevicki and K. Suzuki, Three Dimensional View of the SYK/AdS Duality, JHEP 09 (2017) 017 [arXiv:1704.07208] [INSPIRE].

[10] S.R. Das, A. Ghosh, A. Jevicki and K. Suzuki, Space-Time in the SYK Model, JHEP 07 (2018) 184 [arXiv:1712.02725] [INSPIRE].

[11] A. Almheiri and J. Polchinski, Models of AdS $S_{2}$ backreaction and holography, JHEP 11 (2015) 014 [arXiv: 1402.6334] [INSPIRE]. 
[12] J. Maldacena, D. Stanford and Z. Yang, Conformal symmetry and its breaking in two dimensional Nearly Anti-de-Sitter space, PTEP 2016 (2016) 12C104 [arXiv:1606.01857] [INSPIRE].

[13] J. Engelsöy, T.G. Mertens and H. Verlinde, An investigation of $A d S_{2}$ backreaction and holography, JHEP 07 (2016) 139 [arXiv:1606.03438] [INSPIRE].

[14] J.S. Cotler et al., Black Holes and Random Matrices, JHEP 05 (2017) 118 [Erratum ibid. 09 (2018) 002] [arXiv:1611.04650] [INSPIRE].

[15] G. Mandal, P. Nayak and S.R. Wadia, Coadjoint orbit action of Virasoro group and two-dimensional quantum gravity dual to SYK/tensor models, JHEP 11 (2017) 046 [arXiv: 1702.04266] [INSPIRE].

[16] A. Kitaev and S.J. Suh, The soft mode in the Sachdev-Ye-Kitaev model and its gravity dual, JHEP 05 (2018) 183 [arXiv:1711.08467] [INSPIRE].

[17] G. Sárosi, $A d S_{2}$ holography and the SYK model, PoS (Modave2017) 001 (2018) [arXiv: 1711.08482] [INSPIRE].

[18] T.G. Mertens, The Schwarzian theory - origins, JHEP 05 (2018) 036 [arXiv:1801.09605] [INSPIRE].

[19] P. Saad, S.H. Shenker and D. Stanford, A semiclassical ramp in SYK and in gravity, arXiv: 1806.06840 [INSPIRE].

[20] V. Rosenhaus, An introduction to the SYK model, arXiv:1807.03334 [INSPIRE].

[21] D. Stanford and E. Witten, Fermionic Localization of the Schwarzian Theory, JHEP 10 (2017) 008 [arXiv:1703.04612] [INSPIRE].

[22] V.V. Belokurov and E.T. Shavgulidze, Unusual view of the Schwarzian theory, Mod. Phys. Lett. A 33 (2018) 1850221 [arXiv: 1806.05605] [INSPIRE].

[23] D. Bagrets, A. Altland and A. Kamenev, Sachdev-Ye-Kitaev model as Liouville quantum mechanics, Nucl. Phys. B 911 (2016) 191 [arXiv:1607.00694] [INSPIRE].

[24] D. Bagrets, A. Altland and A. Kamenev, Power-law out of time order correlation functions in the SYK model, Nucl. Phys. B 921 (2017) 727 [arXiv:1702.08902] [INSPIRE].

[25] T.G. Mertens, G.J. Turiaci and H.L. Verlinde, Solving the Schwarzian via the Conformal Bootstrap, JHEP 08 (2017) 136 [arXiv:1705.08408] [INSPIRE].

[26] D.J. Gross and V. Rosenhaus, All point correlation functions in SYK, JHEP 12 (2017) 148 [arXiv: 1710.08113] [INSPIRE].

[27] H.-H. Kuo, Gaussian Measures in Banach Spaces, Springer, Berlin-Heidelberg-NY (1975).

[28] V.V. Belokurov and E.T. Shavgulidze, Exact solution of the Schwarzian theory, Phys. Rev. D 96 (2017) 101701 [arXiv: 1705. 02405] [INSPIRE].

[29] A. Weil, L'intégration dans les groupes topologiques et ses applications, Actual. Scient. et Ind., 869, Paris, Herman (1940).

[30] Yu.A. Neretin, Categories of Symmetries and Infinite-Dimensional Groups, Clarendon Press, Oxford (1996).

[31] L.A. Shepp, Radon-Nikodym Derivatives of Gaussian measures, Ann. Math. Statist. 37 (1966) 321. 
[32] V.V. Belokurov and E.T. Shavgulidze, Extraordinary properties of functional integrals and groups of diffeomorphisms, Phys. Part. Nucl. 48 (2017) 267 [InSPIRE].

[33] E.T. Shavgulidze, Amenability of Discrete Subgroups of Groups of Diffeomorphisms, to be published.

[34] E.T. Shavgulidze, An example of a measure quasi-invariant with respect to the action of a group of diffeomorphisms of the circle, Funct. Anal. Appl. 12 (1978) 203.

[35] E.T. Shavgulidze, A measure quasi-invariant with respect to the action of a group of diffeomorphisms of a finite-dimensional manifold, Sov. Math. Dokl. 38 (1988) 622.

[36] E.T. Shavgulidze, Some Properties of Quasi-Invariant Measures on Groups of Diffeomorphisms of the Circle, Russ. J. Math. Phys. 7 (2000) 464.

[37] A.P. Prudnikov, Yu.A. Brychkov and O.I. Marichev, Integrals and Series. V. 1. Elementary Functions, Gordon Breach, New York-London (1986).

[38] A. Kitaev and S.J. Suh, Statistical mechanics of a two-dimensional black hole, arXiv: 1808.07032 [INSPIRE].

[39] A. Banerjee, A. Kundu and R.R. Poojary, Strings, Branes, Schwarzian Action and Maximal Chaos, arXiv: 1809.02090 [INSPIRE].

[40] H.P. McKean, Stochastic Integrals, Academic Press, NY-London (1969).

[41] S. Lang, $S L_{2}(\boldsymbol{R})$, Addison-Wesley Publishing (1975).

[42] V.A. Smirnov, Feynman Integrals Calculus, Springer, Berlin-Heidelberg-New york (2006). 